\title{
Research Paper: Comparing the Kinematics of the Lower Limb Joints in Jump-Landing Maneuver Among Soccer Players With and Without Histories of Anterior Cruciate Ligament Reconstruction
}

\author{
Moosareza Ghorbani $^{1}$ (D), Farideh Babakhani ${ }^{* *}$ (D)
}

1. Department of Corrective Exercise And Sport Injury, Faculty of Physical Education and Sport Sciences, Allemeh Tabataba'i University, Tehran, Iran.

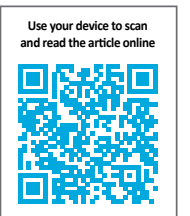

Chation Ghorbani M, Babakhani F. Comparing the Kinematics of the Lower Limb Joints in Jump-Landing Maneuver Among Soccer Players With and Without Histories of Anterior Cruciate Ligament Reconstruction. Physical Treatments. 2020; 10(3):177-184. http://dx.doi.org/10.32598/ptj.10.3.454.1

dol': http://dx.doi.org/10.32598/ptj.10.3.454.1

\section{(i) (5)}

Article info:

Received: 08 Apr 2020

Accepted: 22 Jun 2020

Available Online: 01 Jul 2020

Keywords:

Anterior cruciate

ligament, Fatigue, Soccer, Anterior cruciate ligament reconstruction

\section{A B S T R A C T}

Purpose: Jumping and landing are common activities in soccer, i.e. associated with Anterior Cruciate Ligament (ACL) injuries in the players, especially when experiencing fatigue. The current study aimed to compare the kinematics of the lower limb joints in jump-landing maneuver among soccer players with and without a history of anterior cruciate ligament reconstruction.

Methods: Among active soccer players in the provincial league level in Hamedan Province, Iran, 12 individuals with the intact knee (control group), and 12 individuals with 6 to 24 months post anterior cruciate ligament reconstruction (experimental group) were selected by convivence sampling method as the study participants. During the jump-landing task, the range of motion and the joints' angle at the contact instance were measured before and after performing plyometric fatigue protocol using the Vicon motion capture system. Multivariate Analysis of Variance (MANOVA) at a significance level of $\mathrm{P}<0.05$ was used for intergroup comparisons.

Results: Plyometric fatigue did not affect most of the kinematic variables before and after fatigue experience. Besides, only the hip range of motion revealed a significant difference between the study groups $(\mathrm{P}=0.38)$.

Conclusion: Fatigue had more effect on some variables in individuals with ACL reconstruction, including the left and right hips angles. Moreover, there was a significant difference in some variables between the study groups; the range of motion of the left hip, the contact angle of the right hip before fatigue experience, and the contact angle of the right and left hips after fatigue. In general, a proper rehabilitation program is recommended for athletes with ACL reconstruction to reduce the risk of recurrence of jumping movements in exercise.

\section{* Corresponding Author:}

Farideh Babakhani, PhD.

Address: Department of Corrective Exercise and Sport Injury, Faculty of Physical Education and Sport Sciences, Allemeh Tabataba'i University, Tehran, Iran. Phone: +98 (903) 6452560

E-mail:farideh_babakhani@yahoo.com 


\section{Highlights}

- The plyometric fatigue protocol caused changes in the angles of the lower limb joints, some of which increase the risk of injury to the anterior cruciate ligament.

- Changes in the angles of the lower limb joints increase the risk of knee injuries, especially in healthy footballers.

\section{Plain Language Summary}

Football is a popular sport and has many fans all over the world. Many injuries occur in this sport some of which may cause an athlete to stay away from exercise. One of the most common injuries in football is knee injury, especially anterior cruciate ligament. Factors that can cause damage to this ligament include sudden changes in direction of movement. Excessive force applied to the knee joint and improper biomechanics during landing. Jumping and landing are common activities in most sports, which can increase the risk of knee injuries, however, most injuries have been shown to occur during one-leg landing and reduced knee acceleration. Most athletes decide to have surgery after a ligament rupture, but some athletes express dissatisfaction after reconstruction. Previous studies have shown that there is a possibility of re-injury in these athletes. Well defined that fatigue can increase the risk of injury. Fatigue is also an integral part of football. One of the fatigue protocols that has been used in recent years is the plyometric fatigue protocol, which has been welcomed by trainers due to its ease of implementation and the need for minimal equipment. Since most injuries occur during fatigue, research suggests that comparing them between two groups of healthy athletes and athletes with anterior cruciate ligament reconstruction can yield beneficial results.

\section{I}

\section{Introduction}

$\mathrm{n}$ various sports, when the athlete is involved with jumping and landing maneuvers, like soccer Anterior Cruciate Ligament (ACL) injuries are prevalent [1]. The medical costs of the ACL surgery are very high. In young soccer players, $71 \%-80 \%$ of the injuries occur in the lower extremities [2]. ACL rupture or elongation is very common among these injuries [3]. The most frequent cause of ACL injury in athletes is the non-contact type [1]. Progress in surgical techniques has attracted more attention to performing a post-surgery reconstruction that could improve the knee's stability [4]. In total, 50\% of players fail to return to the tournament after undergoing ACL surgery. A significant percentage of the rest return to play before completing the rehabilitation period due to the team's needs [2]. Furthermore, the medical costs of the ACL are considerable. Athletes who experienced meniscus and other ligaments injuries simultaneously are more prone to be exposed to subsequent injuries, especially knee osteoarthritis [5].

Numerous researchers have investigated the mechanisms of injury occurrence in jumping and landing. Landing from higher altitude, due to high ground reaction force, increases the risk of ACL injuries [6]. It puts too much pressure on the ACL due to kneeling in high valgus position alongside the anterior tibial shear force and high mechanical pressure, whilst jumping [7]. Lower limb muscles could properly absorb and modify the compressive and shear reaction forces (applied to the joints by the ground) by making the movements in the correct way [8]. Fatigue is among the factors that reduce this ability of the muscles [9]. According to the literature, fatigue is a serious risk factor for ACL rupture [7]. Additionally, athletes who underwent cruciate ligament surgery, even after 6 months of rehabilitation, were at lower oxygen consumption levels, compared to healthy individuals [4]. Many methods have been used to investigate the effect of fatigue on post-jump landing operations [10].

Among various exercises, the plyometric ones, which include extravagant contractions and many forces, could easily damage the muscle's anatomy. Furthermore, the high pressure of these exercises could cause fatigue; subsequently, it reduces the force of contraction and the momentum torque [11]. In addition, plyometric exercises have the potential to be well-performed because of the minimal need for equipment, ease of performance, and body weight usage [12]. It is essential to conduct kinematic assessments in the diagnosis of knee injury risk [2]. Accordingly, this method has been used to identify the risk factors in most studies [11]. This is because of the greater impact of fatigue on athletes who have had 


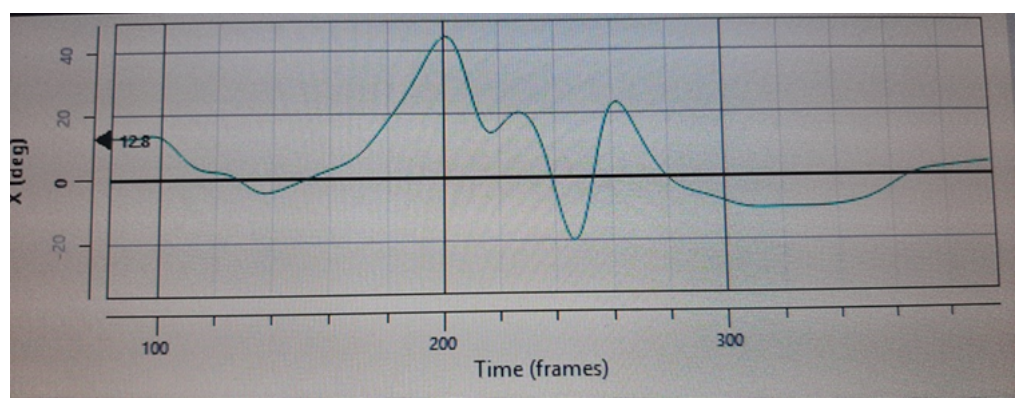

Figure 1. The ankle dorsiflexion angle diagram

PHYSICAL TREA $\$ MENTS

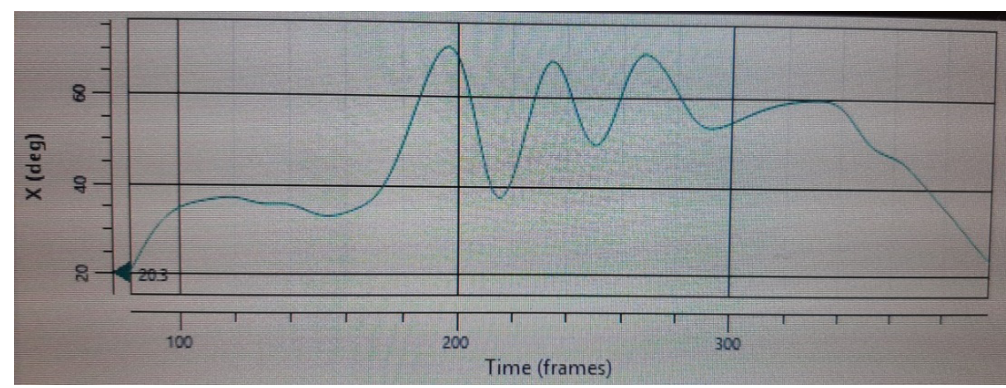

Figure 2. The hip flexion angle diagram

PHYSICAL TREA $\mid$ MENTS

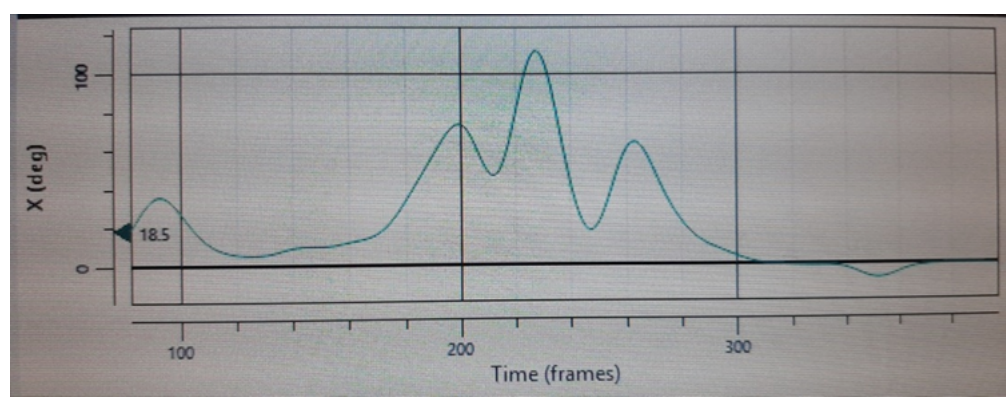

Figure 3. The knee flexion angle diagram

PHYSICAL TREA $\ M E N T S$

ACL reconstruction [13]. The current study compared the kinematics of the lower limb joints in jump-landing maneuver among soccer players with and without histories of ACL reconstruction.

\section{Materials and Methods}

This was a quasi-experimental study with a control group design. The required data were collected in 2020 at Hamadan Azad University. Initially, the target population was 30 soccer players in Hamadan Province, Iran. They aged between 20 and 30 years. Besides, 15 healthy individuals and 15 with ACL reconstruction with hamstring graft were selected by convenience sampling method to participate in this study. Previous studies have investigated the effect of fatigue on lower limb landing biomechanics. Accordingly, it was estimated that a medium to large effect size $(\mathrm{F}=0.3)$ is needed to be detected. With statistical significance set at a two-sided level of 0.05 , a power of 0.8 , and the correlation among repeated measures at 0.5 , a minimum of 10 subjects was required per group.
Large effect size was considered to reflect the strong effect of fatigue on biomechanics observed in previous investigations [14]. Then, based on the stud exclusion criteria, the subjects with clear abnormalities in the body or those with $<6$ months or $>24$ months of ligament reconstruction, and the athletes aged $>30$ or $<20$ years were excluded from the study. Finally, 24 professional soccer players aged 20-30 years with $\geq 8$ years of soccer experience and three training sessions per week were selected as the study participants. The research subjects were assigned into the control $(n=12)$ and experimental $(n=12)$ groups; the control group included the healthy samples and the experimental group consisted of those with ACL reconstruction with a hamstring graft.

Both study groups received appropriate explanations about the research process. Consequently, they provided an informed consent form. The study subjects were assured that their obtained data remains confidential, they could discontinue participating in the study at any time, and that there would be no compulsion to select and con- 
tinue the exercise program. At the beginning of the study, the individual profile form was completed by measuring the height, weight, and length of legs. Other specifications were also completed by the research subjects. The information included the injured leg and the time elapsed since the surgery. Before the test, the study subjects warmed up for 10 minutes to prevent injury. Moreover, the warm-up included slow running and stretching at the lower body [5]. Then, the markers were attached to anatomical landmarks based on the plug-in gait method, including the Anterior Superior Iliac Spine (ASIS), upper posterior, knee lateral epicondyle, the bottom onethird of the thigh, lateral malleolus, second metatarsal's head, and behind the heel bone (calcaneus). Initially, a static calibration trial was recorded [15]. Then, the study subjects were requested to stand at the starting point, i.e. half of the subjects' height to the landing site. Next, the research subjects were advised to jump from a cone to a height of $7.5 \mathrm{~cm}$ located between the starting point and the center of the landing point. They were also explained to land on the specified point after crossing the obstacle with two legs [15]. Using 4 cameras (Vicon peak Ltd., Oxford, UK Vicon), the Range of Motion (ROM), and the contact angle of the hip, knee, and ankle joints were measured. After conducting the pretest, the research subjects performed the fatigue protocol with the plyometric exercise. These exercises included running and jumping in which running comprised high speed running at a dis-

Table 1. The study groups' information

\begin{tabular}{ccc}
\hline & & Mean \\
Variabels & Control & ACL \\
\cline { 2 - 3 } Age $(\mathrm{y})$ & 23.50 & 26.4167 \\
\hline Weight $(\mathrm{kg})$ & 72.5833 & 81.8333 \\
\hline Height $(\mathrm{m})$ & 1.7658 & 1.7867 \\
\hline BMI $(\mathrm{kg} / \mathrm{m} 2)$ & 23.2468 & 25.5110 \\
\hline
\end{tabular}

PHYSICAL TREA $\mid$ MENTS

Table 2. The descriptive findings of the study groups (Mean pre-fatigue data)

\begin{tabular}{|c|c|c|}
\hline \multirow{2}{*}{ Selected Joints } & \multicolumn{2}{|c|}{ Mean $\pm S D$} \\
\hline & Healthy Group & ACL Reconstruction Group \\
\hline Left hip's contact angle & $30.75 \pm 16.48$ & $37.40 \pm 7.21$ \\
\hline Right hip's contact angle & $29.60 \pm 10.43$ & $37.51 \pm 8.93$ \\
\hline Left knee's contact angle & $16.67 \pm 8.08$ & $22.63 \pm 7.96$ \\
\hline Right knee's contact angle & $19.49 \pm 6.16$ & $22.09 \pm 8.76$ \\
\hline Right ankle's contact angle & $-6.29 \pm 6.06$ & $-5.25 \pm 4.89$ \\
\hline Right ankle's contact angle & $-4.10 \pm 6.01$ & $-3.68 \pm 5.24$ \\
\hline Left hip's ROM & $38.09 \pm 12.48$ & $48.79 \pm 11.29$ \\
\hline Right hip's ROM & $41.34 \pm 12.07$ & $47.26 \pm 10.25$ \\
\hline Left knee's ROM & $62.04 \pm 10.37$ & $63.44 \pm 11.53$ \\
\hline Right knee's ROM & $62.57 \pm 13.36$ & $63.65 \pm 13.89$ \\
\hline Left ankle's ROM & $22.51 \pm 15.73$ & $19.59 \pm 9.36$ \\
\hline Right ankle's ROM & $23.94 \pm 16.29$ & $24.50 \pm 5.88$ \\
\hline
\end{tabular}

PHYSICAL TREA MENTS 
Table 3. The descriptive findings of the study groups (Mean after-fatigue data)

\begin{tabular}{|ccc|}
\hline Selected Joints & Healthy Group & Mean \pm SD \\
\hline Left hip's contact angle & $27.99 \pm 8.97$ & ACL Reconstruction Group \\
\hline Right hip's contact angle & $27.86 \pm 8.03$ & $39.85 \pm 8.39$ \\
\hline Left knee's contact angle & $16.25 \pm 7.02$ & $40.30 \pm 9.50$ \\
\hline Right knee's contact angle & $18.36 \pm 7.77$ & $28.68 \pm 21.89$ \\
\hline Right ankle's contact angle & $-5.07 \pm 5.53$ & $22.82 \pm 8.66$ \\
\hline Right ankle's contact angle & $-6.21 \pm 6.81$ & $-3.63 \pm 8.32$ \\
\hline Left hip's ROM & $43.78 \pm 8.43$ & $-2.68 \pm 8.09$ \\
\hline Right hip's ROM & $43.31 \pm 8.75$ & $54.49 \pm 24.19$ \\
\hline Left knee's ROM & $60.03 \pm 11.87$ & $51.15 \pm 18.16$ \\
\hline Right knee's ROM & $56.70 \pm 17.77$ & $65.82 \pm 15.67$ \\
\hline Left ankle's ROM & $27.27 \pm 21.39$ & $62.99 \pm 13.74$ \\
\hline Right ankle's ROM & $17.66 \pm 11.47$ & $18.65 \pm 7.08$ \\
\hline
\end{tabular}

PHYSICAL TREA M MENTS

tance of 10 meters in the form of sweeping and jumping (i.e. double-leg jumping \& landing, single-leg jumping \& landing, vertical jumping, jumping over obstacles, tuck jumping, \& single-leg jumping between lines), i.e. performed in three sets of 10 meters; between each set, 30 seconds was considered for resting [16]. Vicon nexus software was used to analyze the data, which is shown in the top-sample diagrams on the sagittal plate (Fiures $1,2,3)$. Immediately after conducting the fatigue protocol, the posttest trials were recorded. The obtained data were analyzed using Multivariate Analysis of Variance (MANOVA). All of the evaluations were conducted at the significance level of 0.05 in SPSS.

\section{Results}

The study groups' information, such as body weight, height, and Body Mass Index (BMI) are summarized in the table below (Table 1).

According to the Shapiro-Wilk test data and the normal distribution of the obtained data ( $\mathrm{P}>0.05)$, MANOVA was used to compare between-group differences concerning weight $(\mathrm{P}=0.101)$ height $(\mathrm{P}=0.486)$, and $\mathrm{BMI}(\mathrm{P}=0.107)$. There was no significant difference between the study groups in terms of the above-mentioned variables.
The achieved results revealed a significant difference between the maximal pre-fatigue range of motion in the left hip joint $(\mathrm{P}=0.38)$. Besides, the same value in the left hip of the affected group was approximately $9.6 \mathrm{de}$ grees higher; however, respecting ROM in the right hip, there was no significant difference between the study groups $(\mathrm{P}=0.222)$. There was no difference in the $\mathrm{ROM}$ in the left $(\mathrm{P}=0.757)$ and right $(\mathrm{P}=0.848)$ knee joints as well as the left $(\mathrm{P}=0.587)$ and right $(\mathrm{P}=0.913)$ ankles. After fatigue experience, the ROM difference was not significant in either joint [left hip $(\mathrm{P}=0.162)$, right hip $(\mathrm{P}=0.192)$, left knee $(\mathrm{P}=0.319)$, right knee $(\mathrm{P}=0.343)$, left ankle $(\mathrm{P}=0.199)$, right ankle $(\mathrm{P}=0.611)]$. There was no significant difference between the control and test groups concerning the joint angles at the moment of heel contact before fatigue, except for the right hip $(\mathrm{P}=0.049)$. However, after fatigue experience, at the moment of contact between left $(\mathrm{P}=0.03)$ and right $(\mathrm{P}=0.02)$ hip, there was a significant difference between the study groups. In the left hip, that was about $11.86^{\circ}$ and in the right hip about $12.44^{\circ}$ higher in the experimental group, compared to the controls. The descriptive findings are summarized in (Table $2 \& 3$ ).

\section{Discussion}

The present study results indicated that fatigue impacted some of the kinematic parameters after the jump in the 
studied subjects. However, in some joints, there was no difference between the study groups. According to Sohel et al., the control group performed a landing strategy by reducing knee flexion; their study revealed that knee flexion is critical for absorbing forces, and reducing knee flexion during landing is a risk factor for a knee injury [17].

In the present study, the knee impact angle decreased during landing in the control group after fatigue experience; however, this change was not observed in the experimental group. In another study involving 19 testers as an example, the effect of cognitive and neuromuscular fatigue on lower extremity biomechanics was investigated. The relevant results suggested that knee flexion decreased after fatigue experience. Besides, the fatigue of the lower extremity significantly altered the kinematics of the knee. These changes occurred among female non-elite athletes and no one could expect that to be the case in elite athletes. However, fatigue does not always decrease knee flexion angle; some studies have reported that fatigue increases knee flexion $[18,19]$.

In the present study's experimental group, this result was also established and fatigue increased the flexion angle in this group. Fatigue was not measured in this study either, and perhaps at actual levels, the fatigue variations were different. Previous studies have indicated that $50 \%$ of fatigue changes belong to the biomechanical alternations of landing; therefore, it is not important to determine fatigue levels [20]. Acknowledging that fatigue affects muscle strength and increases cruciate ligament injury is not always accurate. Moreover, some investigations have reported no difference in ACL injury in the first or second halves of the season. Concerning kinematic and kinetic studies, most of the force plate and three-dimensional motion systems are used. Besides, in some studies, tired athletes who look to the ground (landing point) appear to have a higher flexion angle in the thigh and knee; it reduces the risk of damage to the cruciate ligament [7].

In the present study, the explored individuals were also familiar with the jump environment and the size and height of the obstacle before jumping; thus, such familiarity may be a reason for the non-significant changes in knee and hip angles. In another study, testers were used, and 15 to 19 months were passed since their reconstruction. The obtained results revealed that contrary to most hypotheses, fatigue in lower extremity biomechanics was not significantly different in those with cruciate ligament reconstruction. Additionally, the collected data justified the conclusion that individuals with ACL reconstruction, usually rely on a healthy leg in their movements. Subsequently, this could generate fatigue in the leg and the rest of the body will remain unchanged. Therefore, individuals with cruci- ate ligament reconstruction may have used compensatory motor patterns before fatigue. They also do not need further compensation in response to neuromuscular fatigue. Those who have undergone reconstruction may also have used a standard protocol for rehabilitation. Moreover, these differences could certainly affect one's strength and biomechanics characteristics [21].

The present study sample was also composed of professional soccer players; therefore, it is unlikely that they have used a specific rehabilitation program. The vertical ground reaction force is altered by knee, thigh, and ankle plantarflexion, assuming that a smoother and faster landing reduces the adjustment capacity of the force. As a result, it increases the forces as well as the risk of injury [8]. In the present study, even minor changes in the angle of the joints could cause slight changes in the forces. In another study, 10 women and 10 men were explored as testers to investigate the reaction force and kinematics of the joints. The fatigue protocol was a comparison of knee flexion and extension movements. The collected results signified that knee extension fatigue training significantly reduced the ground reaction force. Furthermore, individuals with greater flexion angle landed on the knee; however, knee flexion fatigue did not affect the ground reaction force [9].

Accordingly, plyometric fatigue does significantly influence the kinematics of healthy and injured individuals; although the statistical sample underlying the fatigue protocol and gender may be affected. The present study limitations included the lack of investigating females and the lack of EMG activity record before and after fatigue. Furthermore, the effect of fatigue induced by plyometric exercises was investigated; different protocols and communities cold provide different results. The use of an EMG device is recommended to evaluate the activity of the quadriceps and hamstrings muscles. Moreover, women have a higher risk of ACL injury than men; thus, using women in this study could have provided beneficial results.

\section{Conclusion}

The obtained data suggested that plyometric fatigue did not cause numerous changes in the ROM of the lower limb joints in the study groups. The contact angle in the lower limb joints did not significantly change in the study groups. These results signified that plyometric fatigue did not increase the damage to the lower limbs, even in athletes who had reconstructed anterior cruciate ligament. 


\section{Ethical Considerations}

\section{Compliance with ethical guidelines}

This study was approved by the Ethics Committee of the University of Sports Science Research Institute (IR.SSRI. REC.1399.749).

\section{Funding}

This article was extracted from a MA thesis of Moosareza Ghorbani, Department of Corrective Exercise And Sport Injury, Faculty of Physical Education and Sport Sciences, Allemeh Tabataba'i University.

\section{Authors' contributions}

All authors equally contributed in preparing this article.

\section{Conflict of interest}

The authors declared no conflict of interest.

\section{References}

[1] Åman M, Larsén K, Forssblad M, Näsmark A, Waldén M, Hägglund M. A nationwide follow-up survey on the effectiveness of an implemented neuromuscular training program to reduce acute knee injuries in soccer players. Orthopaedic Journal of Sports Medicine. 2018; 6(12):2325967118813841. [DOI:10.1177/2325967118813841] [PMID] [PMCID]

[2] Read PJ, Oliver JL, De Ste Croix MBA, Myer GD, Lloyd RS. Landing kinematics in elite male youth soccer players of different chronologic ages and stages of maturation. Journal of Athletic Training. 2018; 53(4):372-8. [DOI:10.4085/10626050-493-16] [PMID] [PMCID]

[3] Fulstone D, Chandran A, Barron M, DiPietro L. Continued sex-differences in the rate and severity of knee injuries among collegiate soccer players: The NCAA injury surveillance system, 2004-2009. International Journal of Sports Medicine. 2016; 37(14):1150-3. [DOI:10.1055/s-0042-112590] [PMID]

[4] de Almeida AM, Silva PRS, Pedrinelli A, Hernandez AJ. Aerobic fitness in professional soccer players after anterior cruciate ligament reconstruction. PLoS One. 2018; 13(3): e0194432. [DOI:10.1371/journal.pone.0194432] [PMID] [PMCID]

[5] McHugh MP, Tyler TF, Mirabella MR, Mullaney MJ, Nicholas SJ. The effectiveness of a balance training intervention in reducing the incidence of noncontact ankle sprains in high school football players. The American Journal of Sports Medicine. 2007; 35(8):1289-94. [DOI:10.1177/0363546507300059] [PMID]

[6] Lucci S, Cortes N, Van Lunen B, Ringleb S, Onate J. Knee and hip sagittal and transverse plane changes after two fatigue protocols. Journal of Science and Medicine in Sport. 2011; 14(5):453-9. [DOI:10.1016/j.jsams.2011.05.001] [PMID] [PMCID]
[7] Sandon A, Engström B, Forssblad M. High risk of further ACL injury in a 10-year follow-up study of ACL-reconstructed soccer players in the Swedish National Knee Ligament Registry. Arthroscopy. 2020; 36(1):189-95. [DOI:10.1016/j. arthro.2019.05.052] [PMID]

[8] Pflum MA, Shelburne KB, Torry MR, Decker MJ, Pandy MG. Model prediction of anterior cruciate ligament force during drop-landings. Medicine and Science in Sports and Exercise. 2004; 36(11):1949-58. [DOI:10.1249/01. MSS.0000145467.79916.46] [PMID]

[9] Enoka RM, Duchateau J. Muscle fatigue: What, why and how it influences muscle function. The Journal of Physiology. 2008; 586(1):11-23. [DOI:10.1113/jphysiol.2007.139477] [PMID] [PMCID]

[10] Chavez A. The Effect of Fatigue on ACL Injury Risk in the Athletic Population. 2012.

[11] Drinkwater EJ, Lane T, Cannon J. Effect of an acute bout of plyometric exercise on neuromuscular fatigue and recovery in recreational athletes. The Journal of Strength \& Conditioning Research. 2009; 23(4):1181-6. [DOI:10.1519/ JSC.0b013e31819b79aa] [PMID]

[12] Egan-Shuttler JD, Edmonds R, Eddy C, O'Neill V, Ives SJ. The effect of concurrent plyometric training versus submaximal aerobic cycling on rowing economy, peak power, and performance in male high school rowers. Sports MedicineOpen. 2017; 3(1):7. [DOI:10.1186/s40798-017-0075-2] [PMID] [PMCID]

[13] Gallo MC, Bolia IK, Jalali O, Rosario S, Rounds A, Heidari KS, et al. Risk factors for early subsequent (revision or contralateral) acl reconstruction: A retrospective database study. Orthopaedic Journal of Sports Medicine. 2020; 8(2):2325967119901173. [DOI:10.1177/2325967119901173] [PMID] [PMCID]

[14] Webster KE, Santamaria LJ, McClelland JA, Feller JA Effect of fatigue on landing biomechanics after anterior cruciate ligament reconstruction surgery. Medicine \& Science in Sports \& Exercise. 2012; 44(5):910-6. [DOI:10.1249/ MSS.0b013e31823fe28d] [PMID]

[15] Butler RJ, Russell ME, Queen R. Effect of soccer footwear on landing mechanics. Scandinavian Journal of Medicine \& Science in Sports. 2014; 24(1):129-35. [DOI:10.1111/j.16000838.2012.01468.x] [PMID]

[16] Chmielewski TL, GeorgeSZ, TillmanSM, Moser MW, Lentz TA, Indelicato PA, et al. Low-versus high-intensity plyometric exercise during rehabilitation after anterior cruciate ligament reconstruction. The American Journal of Sports Medicine. 2016; 44(3):609-17. [DOI:10.1177/0363546515620583] [PMID]

[17] Sohel AA. Effect of Arm Motion on Standing Lateral Jump Performance. 2017.

[18] Kunugi S, Masunari A, Yoshida N, Miyakawa S. Association between cumberland ankle instability tool score and postural stability in collegiate soccer players with and without functional ankle instability. Physical Therapy in Sport. 2018; 32:29-33. [DOI:10.1016/j.ptsp.2018.03.002] [PMID]

[19] Peh ECY, Liang Y-C, Guan YL, Zeng Y. Optimization of cooperative sensing in cognitive radio networks: A sensing-throughput tradeoff view. IEEE Transactions on 
Vehicular Technology. 2009; 58(9):5294-9. [DOI:10.1109/ TVT.2009.2028030]

[20] Aizawa J, Ohji S, Koga H, Masuda T, Yagishita K. Correlations between sagittal plane kinematics and landing impact force during single-leg lateral jump-landings. Journal of Physical Therapy Science. 2016; 28(8):2316-21. [DOI:10.1589/ jpts.28.2316] [PMID] [PMCID]

[21] Thomas AC, Lepley LK, Wojtys EM, McLean SG, Palmieri-Smith RM. Effects of neuromuscular fatigue on quadriceps strength and activation and knee biomechanics in individuals post-anterior cruciate ligament reconstruction and healthy adults. Journal of Orthopaedic \& Sports Physical Therapy. 2015; 45(12):1042-50. [DOI:10.2519/ jospt.2015.5785] [PMID] [PMCID] 